\title{
Charting the Course to Universal Health in the Americas: Cristian Morales PhD, PAHO/WHO Representative in Cuba
}

\author{
Gail Reed MS
}

\begin{abstract}
After leaving Chile during the Pinochet era, Dr Morales studied economics, health administration and international health at the University of Montreal. But his baptism in the field came in Haiti, where he was first PAHO advisor to the health ministry, and then for five years was responsible for human resources and health economics in the $\mathrm{PAHO}$ offices in the capital of Port-au-Prince. He was at his post during the flooding in Gonaïves, five hurricanes, the 2010 earthquake and the ensuing cholera epidemic-doubtless the most dramatic and complex times for the country's health in recent history. Before becoming the PAHO/WHO Representative in Cuba in 2015, he was Regional Advisor in Financing and Health Economics based in Washington, DC. In that role, he plunged into the often thorny debates about just how far governments of the Americas were willing to go towards achieving universal health-universal coverage plus universal access. The result was a historic resolution passed in late 2014 by PAHO's Directing Council (CD53.R14 Strategy for Universal Access to Health and Universal Health Coverage). Dr Morales talks about the process, the outcomes... and the road ahead.
\end{abstract}

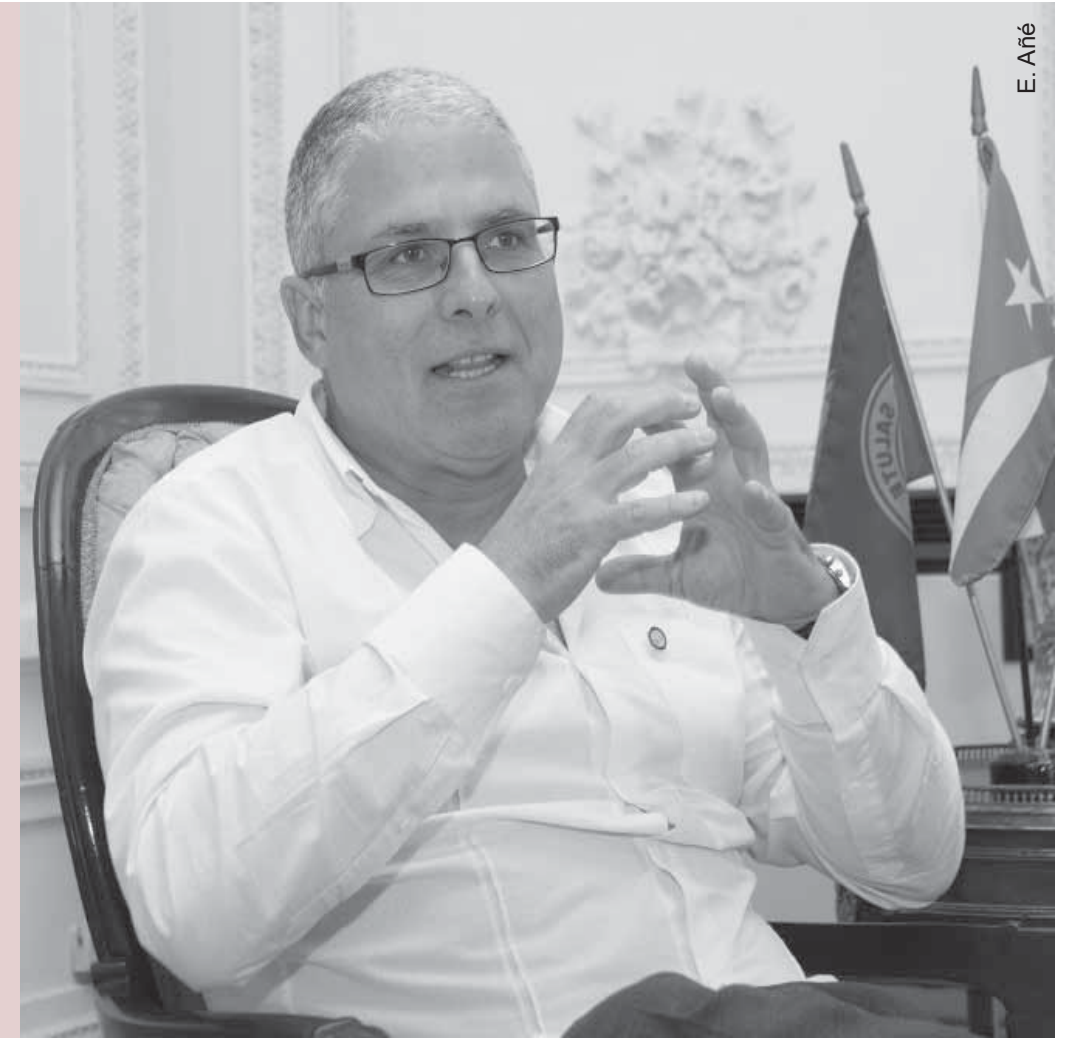

MEDICC Review: Universal health coverage is a concept whose ambiguity has led to different interpretations, and hence different approaches to health system reforms. What motivated the most recent PAHO discussions and the Resolution?

Cristian Morales: They began as part of a global debate on universal health coverage (UHC) that started much earlier, crystalizing around WHO's 2010 World Health Report. Here in the Americas, we needed to clarify our intentions as a region, to profoundly analyze and commit to ensuring health for all in a realistic framework, meaning not only universal coverage but also universal access to quality health services... in short, universal health (a term that finally came into use in July of 2015). As a regional body, we were looking to make a clear statement and develop a workable strategy for countries in our region to reach that goal.

We began to conceptualize access not only as full access to health services, including the absence of barriers, whether geographic, financial, cultural or any other kind; but also access to policies that address the social determinants of health, public policies that promote and strengthen health. In this context, universal coverage provides the means to reach universal access, encompassing the breadth and quality of services at all levels, health institutions, technologies, financing and human resources. This vision poses primary health care as the pillar of services, but includes every level of care. This approach demands integrated, quality services that promote health and are preventive, curative, rehabilitative and palliative. It rejects the notion of a "minimum package" of services in favor of integrated, comprehensive care.

MEDICC Review: Where does the road to universal health begin?

Cristian Morales: Nothing can be done without political will. Universal health has to be embraced by each nation as an essential element of public policies based on the right to health, with a larger goal of creating equity. Achieving universal health involves much more than just the health sector.

In practice, this presents a different way of prioritizing scarce resources since universal health is based on the principle of the right to health, regardless of ability to pay. It implies a process for each country in which services expand-for everyone across the boardgiven the possibilities at hand. We see the examples of Cuba, Canada and Brazil, on the one hand, countries that have public systems responding to this principle. But their models aren't the only ones. With the tremendous diversity in the Americas' health systems, we can't focus on reaching universal health with a single model.

At the same time, we are interested in what each health system can produce for its population, and in seeing that financial barriers 
do not impede access to health care. You have to recall that the number one cause of personal bankruptcy in the United States, for example, is out-of-pocket health expenditures.

For universal health to become a reality in our region, we need systems of financing that break segmentation and fragmentation, and in which equity, broad access and service integration are explicit.

MEDICC Review: Financing has been suggested as the Achilles' heel of expanding health access and coverage and to reaching universal health. What changes need to be made to make it feasible?

Cristian Morales: The PAHO Resolution marked a milestone because it recognizes financing as a necessary condition to implement universal health strategies: health must be a priority for the whole society, and thus a funding priority, where monies are apportioned efficiently. It established $6 \%$ of GDP as a benchmark for public expenditures on health, which is significant, given that for the last few years average health spending in the region has been only about $4 \%$.

In the past, we've seen investments grow in the private sector, but now this must happen in the public sector, in order to direct investments where they can meet the most needs, particularly among vulnerable groups. These kinds of investments are the ones that decrease inequities, quite intentionally so, which is not the raison d'être of the private sector. Governments are the ones to make equity a priority, to make health care more person- and community-centered, and for this change in the status quo, you need targeted investment in the context of greater priority for health in public spending.

Financing, in many cases, will depend on pooling resources from many plans or sectors, which is more efficient. Why? Because the larger the pool of people, the lower the cost of care. The pool also needs diversity: rich and poor, young and old, by categories of employment and health status, etc. The important thing is to integrate resources, so that contributions from one portion of the pool open doors to services for other portions that might not otherwise receive them. One way to achieve this is by levying progressive taxes on the whole population, but of course that's only one way.

\section{MEDICC Review: Can you give us examples of progress in financing schemes?}

Cristian Morales: Yes. In Chile, you have two insurance regimes: one private and one public. Up to $15 \%$ of the population has private insurance, while some $83 \%$ has public insurance. The AUGE (Universal Access with Explicit Guarantees) regime was introduced in 2005, intended to oblige all insurers to offer the same opportunities and service guarantees. The recommendation is to eventually fuse the two insurance schemes into one system, leaving private sector services to complement public ones only where necessary. And financing then would be in a single pool, as you see in Uruguay.

This would improve efficiency of health system financing, and also intentionally create a large, diverse pool to help address equity, so that contributions by the rich are re-apportioned towards services for the poor and contributions by the healthy re-apportioned towards those who are ill.
There is a space for the private or for-profit health sector in the Americas. But what is of concern is when society's inequities are aggravated by private-sector participation in financing schemes for health services. Out-of-pocket expenses are already significant in the Americas, contributing to disparities: they simply condemn people to poverty. This applies especially to those who live just above the poverty line, who are vulnerable to catastrophic health expenses. Sooner or later, they will fall back into poverty... and never emerge. We need to fight against this kind of inequity by not using direct payments, out-of-pocket expenditures, to finance health systems.

The OECD countries with the best results in satisfying population health needs spend over $6 \%$ of GDP on health (some up to $8 \%$ in public expenditures), while well under $20 \%$ of health expenses are out-of-pocket. Among the 6 to 8 countries in the Americas with less than $20 \%$ direct payments (which I would call "impoverishing payments") is Cuba.

"Universal access to health and universal health coverage imply that all people and communities have access, without any kind of discrimination, to comprehensive, appropriate and timely, quality health services determined at the national level according to needs, as well as access to safe, effective, and affordable quality medicines, while ensuring that the use of such services does not expose users to financial difficulties, especially groups in conditions of vulnerability. Universal access to health and universal health coverage require determining and implementing policies and actions with a multisectoral approach to address the social determinants of health and promote a society-wide commitment to fostering health and well-being."

- Introduction to the PAHO Directing Council Resolution, Strategy for Universal Access to Health and Universal Health Coverage, 2014

MEDICC Review: The PAHO Resolution also calls for each country to develop a Plan of Action to achieve universal health. What is the progress on this score?

It is essential that-depending on the possibilities, history, human resources and culture-each country forge its own path as quickly as possible to universal health through a Plan of Action. Several countries already have these, including Canada, Cuba, Uruguay, Costa Rica and Ecuador, among others.

The idea is to focus on where change is needed in four strategic areas: models of care and services organization to expand access to integrated quality services; health system stewardship and governance strengthening; financing that improves equity and efficiency, working towards elimination of direct payments; and intersectoral work, coordinated to address social determinants of health. The point is to advance comprehensively. But of course not everything can be done at once; a particular country's situation might mean that models of care can be tackled first, then other aspects later, depending on what is politically and economically feasible. The first step is understanding the key problems in each area.

One important concept is differentiated services. While these plans propose universal access, they also have to address different 
needs. For example, indigenous peoples require a different response from health systems: in Panama, their infant mortality is five times the national rate. Indigenous populations face similar situations in Venezuela, Canada and Chile, too. Inequality is at the core of these differences and thus society and health systems bear a different and greater responsibility towards these populations.

As a regional organization, we're making inroads validating the monitoring and evaluation framework of the country Plans of Action. A number of nations, including Cuba, have been working on this. The objective is not to "rank" countries, but rather for governments to have the instruments and indicators to assess their own progress. Our goal is to inform decision-making in each country, be of service to each, and appraise achievements.

MEDICC Review: How is progress towards universal health being measured, and how does this process relate to the UN's Sustainable Development Goals (SDGs)?

Cristian Morales: We are monitoring progress in terms of immediate, intermediate and long-term results. The first has to do with adopting the country Plans of Action to concretize the regional strategy. These plans should include measurable actions, and factors to leverage new models of care, reinforce government authority and intersectoral programs to address the social determinants of health. The intermediate results will reflect the capacity to structure universal health, to protect the population against poverty related to direct payments, effective access to health care, etc. The long-term results will be indicated by effectiveness of national health systems in improving health and equity.

In this context, universal health is part and parcel of more sustainable development. If you look at the SDGs, you'll find they're in sync with PAHO's approach. For example, SDG goal 3 for education and health explicitly includes universal health services in 6 objectives.

Improving health helps build a more cohesive society, diminishes conflicts, and improves the economic and business climate. To the extent that health equity is achieved, a more equitable society is also achieved, increasing capacities for human beings' development and productivity in the broadest sense. Thus, universal health is our grain of sand in building sustainable human development. The right to health itself has to be fought for, but once achieved, will be hard to dismantle.

MEDICC Review: Social medicine has strong roots in Latin America. How does it relate to your understanding of universal health?

Social medicine is based on values and particularly on the right to health. In fact, 25 countries in the region include this right in their constitutions. Social medicine defends the concepts of equity and solidarity; it perceives everyone as equals, as compatriots, whether they are citizens or immigrants. It rejects the notion that people should suffer the consequences of health systems that replicate their societies' inequities. This ethic of solidarity is reflected in suggestions for health financing mechanisms, in which those who have more pay more to achieve more equitable resource redistribution.

This long tradition of the struggle for health and of social medicine in our region makes an important contribution to changing models of health care themselves, from hospitals to community-based
"This generation will no longer tolerate a lack of access to health services to meet their health needs. Universal health is a right, not a privilege, and it's smart-like any good investment."

—Dr Carissa Etienne, PAHO Director, December 9, 2015

services, developing horizontal networks, extramural training and practice, just to mention a few areas.

MEDICC Review: You had experience in Cuba before your current posting. How do you see Cuba's role in the region's path to universal health?

Cuba has to continue contributing its experience and expertise to this regional strategy. It's enough to look at expenditures and outcomes on the island. The country has outcomes similar to those of Canada, with fewer resources, indicating well-directed public expenditures.

Cuba has one of the Americas' most progressive approaches to health: intersectorality was begun long ago with the national literacy campaign; health governance is another virtue; and broad access to services is an experience worth sharing. In addition, the health system has managed to integrate natural and traditional medicines from indigenous and other cultures. This is an important lesson, since we need to be open to the world beyond Western medicine. It would be very healthy for others in the region to see this example in Cuba, particularly because we need to recover and value the contribution of indigenous cultures to health and medicine.

The country continues to build its health system, facing different and more complex health issues. For example, population aging is an important factor: longer life expectancy is an achievement, but is complex and represents a challenge, including more non-communicable diseases. The country also faces infectious diseases, requiring new approaches. New technologies need to be evaluated to incorporate them effectively.

In general, the country is adapting to changing and very demanding times. So in Cuba as everywhere, improvements are needed in organization of services and in making them correspond to the specific needs of specific populations, in the context of integrated service delivery. Regulatory aspects and the social role of health as a contributor to development are well understood. Some of the challenges have to do with improving the conditions for all workers in Cuba. The goals now, and there is leadership for this, are to spend more efficiently and to achieve greater quality in all services for the whole population.

They are improving through innovation, motivated by their own dissatisfaction. In fact, one of Cuba's great strengths is innovation. Take a look at the biotech industry, which can contribute well beyond Cuba. That industry developed under the extremely difficult conditions of the 1990s, amidst economic disaster and the US embargo. They were able to transform theory into practice, and develop technologies to save lives. Progress in genetics and genetic counseling available in primary health care-including for pregnant women-is another example, facilitating risk identification.

What there is in Cuba is great dedication to solidarity and to people's health. -1 . 